\title{
Le développement des services stratégiques aux entreprises et le développement économique régional : le cas de la région de la Capitale- Nationale du Québec
}

\author{
Prosper Habimana \\ Doctorant en développement régional \\ Serge Côté \\ Centre de recherche sur le développement territorial (CRDT) \\ Université du Québec à Rimouski
}

\section{INTRODUCTION}

Les services stratégiques aux entreprises (SSE) font partie de la grande famille du secteur tertiaire qui regroupe toutes les activités de services, c'est-à-dire tout ce qui ne se classe pas dans les secteurs primaire et secondaire. L'importance croissante du

\section{DÉFINITIONS ET TYPOLOGIES}

Les services sont considérés depuis longtemps comme occupant une place majeure dans l'évolution de l'économie contemporaine (entre autres, Fourastié, 1949; Fuchs, 1968). La composition de la grande famille des services est fort diversifiée et inclut toute une gamme d'industries. Plusieurs classifications des activités de services ont été développées par différents auteurs dont Browing et Singelmann (1978), Martinelli (1991), Moyart (2006a).

Pour Browing et Singelmann (1978), les activités de services peuvent se classer en quatre groupes : les services aux producteurs (finance, assurances, services intermédiaires aux entreprises); les services de distribution (transports et communications, commerce de gros et de détail); les services sociaux (services de santé, postes, éducation, gouvernement, bien-être) et les services personnels (services personnels et domestiques, hôtels et restaurants, réparations, détente).

Selon Martinelli (1991), les activités de services peuvent être regroupées dans les quatre catégories suivantes : l'infrastructure sociale (justice, sécurité sociale, associations et organisations profession- secteur tertiaire dans le développement économique des pays et des régions depuis les années 1970 a motivé plusieurs chercheurs à s'intéresser aux activités de ce secteur.

nelles, syndicales et internationales, santé, éducation et autres services sociaux); les services aux consommateurs (commerce de détail, hôtels et restaurants, réparations, services culturels et récréatifs, services personnels); l'infrastructure de distribution (banque et finance, commerce de gros et intermédiaires de distribution, transports, [télé]communications) et les services aux entreprises (assurances, immobilier, laboratoires de recherche et développement [R-D], services juridiques, services de conseils professionnels et techniques, marketing, publicité, relations publiques et services de conférence, services de photocopie et dactylographie, services de nettoyage et gardiennage, réparation et maintenance de biens de production).

Moyart (2006a) regroupe, elle aussi, les activités de services en quatre catégories: les services aux entreprises (comptabilité, ingénierie, intérim, publicité, etc.); les services aux ménages (hôtels et restaurants, soins, loisirs, etc.); les services d'intérêt collectif (santé, éducation, défense, etc.) et les services de distribution, d'intermédiation ou d'intégration (commerce, transports, communications, etc.). 
Toutes ces classifications témoignent d'une grande hétérogénéité dans les activités de services. Par ailleurs, une des caractéristiques majeures qui en ressort est la distinction entre les services aux entreprises et les services aux ménages (Moyart, 2006a). Ceci nous amène à définir de façon simple les services aux entreprises comme étant les services rendus aux entreprises et organisations, c'est-à-dire les services fournis par des entités privées, publiques et parapubliques aux autres entreprises et organisations pour leur permettre de poursuivre leurs activités.

\section{Ceci nous amène à définir de façon simple les services aux entreprises comme étant les services rendus aux entreprises et organisations, c'est-à-dire les services fournis par des entités privées, publiques et parapubliques aux autres entreprises et organisations pour leur permettre de poursuivre leurs activités.}

Les SSE sont une composante des services aux entreprises. En effet, compte tenu de leur rôle, certains services aux entreprises sont définis comme des services banals, alors que d'autres sont considérés comme revêtant une importance particulière pour les entreprises et comme source de croissance économique. Ces derniers sont désignés dans la littérature comme des services de haut niveau aux entreprises (Zuliani, 1995, cité par Moyart, 2006b), des services aux entreprises à haut niveau de connaissance (Camacho et Rodriguez, 2006; Shearmur et Doloreux, 2009), des services avancés aux entreprises (Nagy, 2006), des services spécialisés aux entreprises (Dansereau et Polèse, 1977), des services supérieurs aux entreprises (Martin, 1986; Polèse, 1986; Polèse et Shearmur, 2005), etc. Nous retiendrons dans le présent article l'appellation de services stratégiques aux entreprises (SSE) empruntée à l'Organisation de coopération et de développement économiques (OCDE) (OCDE, 1999). Nous reviendrons sur leur rôle dans la section suivante. Parmi les caractéristiques des SSE soulignées par de nombreux auteurs, on trouve le fait qu'il s'agit : de services qui sont exportables ou aident des entreprises à réaliser des activités exportables et à diminuer leurs importations; de services qui contribuent à l'avancement des entreprises utilisatrices en leur permettant de se différencier par la réalisation d'activités comme les études de marché, la planification des opérations, l'introduction d'innovations et la gestion de l'information; ou encore de services qui soutiennent dans le court, moyen ou long terme la performance et la compétitivité des entreprises. Il n'existe pas de liste de SSE qui fasse l'unanimité et qui soit exhaustive. Toutefois, tous les auteurs semblent tomber d'accord pour dire que les SSE sont des services à haut niveau de savoir. Nous nous limiterons dans cet article à une dizaine de types de services choisis à partir de deux sources : notre recherche dans la région de la Capitale-Nationale du Québec $^{1}$ et la contribution de Shearmur et Doloreux (2009). Selon les résultats de notre recherche, les principaux SSE pour les établissements de cette région sont les services financiers, de R-D et d'innovation, d'informatique, de communication, de conseils scientifique, techniques et en gestion, de génie et de marketing. Nous y ajoutons les services juridiques, d'architecture et d'autres services scientifiques, techniques et professionnels, qui sont aussi considérés, de même que ceux cités précédemment, comme des services aux entreprises à haut niveau de connaissance (knowledge-intensive business services) dans la liste dressée par Shearmur et Doloreux (2009). Cette façon de désigner les activités choisies est fidèle dans les grandes lignes aux appellations en usage dans la version $2003 \mathrm{du}$ Système de classification des industries de l'Amérique du Nord (SCIAN).

\section{RÔLE DES SSE DANS LE DÉVELOPPEMENT RÉGIONAL}

Le secteur tertiaire en général et les services aux entreprises en particulier ont suscité l'intérêt des chercheurs en raison du ralentissement de la croissance industrielle et la montée des services en matière de création d'emploi.
Les activités du secteur tertiaire ont longtemps été considérées comme des activités induites qui ne peuvent pas jouer de rôle actif dans le développement, suivant la vision industrialiste selon laquelle elles suivent passivement la localisation des industries agricole et manufacturière. Cette vision a longtemps guidé les politiques de 
développement régional qui ont été orientées vers le soutien des industries primaire et secondaire et leur localisation dans les régions, oubliant ainsi le secteur tertiaire (Polèse, 1986 ; Gallouj et al., 2006). À partir du milieu des années 1970 et surtout au début des années 1980, le secteur tertiaire en général et les services aux entreprises en particulier ont suscité l'intérêt des chercheurs en raison du ralentissement de la croissance industrielle et la montée des services en matière de création d'emploi (Gallouj et al., 2006).

Les recherches réalisées ces dernières années montrent que les services aux entreprises jouent un rôle très actif dans la performance des entreprises et dans le développement régional, et ce, de différentes façons : (1) ils participent activement dans la création d'emploi; (2) ils s'exportent et permettent une augmentation des revenus au niveau local; (3) ils induisent un effet de compétitivité - productivité dans les entreprises régionales qui les utilisent; (4) ils ont une capacité d'attirer d'autres activités à s'implanter dans la région; et, (5) ils jouent un rôle déterminant dans la recomposition des régions en difficulté (Moyart, 2006b).

Avant de détailler les différents aspects du rôle des services aux entreprises dans le développement des régions, signalons que ces services ne se distribuent pas uniformément dans l'espace. Ils ont en effet tendance à se concentrer dans les zones métropolitaines. Le lien que certains auteurs font entre le dynamisme économique, représenté par exemple par la capacité d'innovation, et les services aux entreprises laisse entendre que les régions mieux dotées en services, comme les métropoles, disposent d'un potentiel d'innovation supérieur aux autres et qu'en général, quoiqu'il puisse y avoir des exceptions, l'innovation va en diminuant à mesure que l'on s'éloigne des métropoles (Shearmur, 2010). Dans une région comme celle de la CapitaleNationale du Québec, qui comprend un noyau métropolitain et une vaste ceinture rurale, il y aura lieu de nous demander dans quelle mesure l'innovation pourra éclore et produire ses effets dans toutes les parties du territoire. Nous reviendrons sur ce sujet dans la conclusion de l'article. Il est maintenant temps de considérer les différentes manières dont les services aux entreprises contribuent au développement des régions.

\subsection{Services aux entreprises et création d'emplois}

De nos jours, le secteur tertiaire est le chef de file en matière de création d'emplois dans de nombreux pays et régions, et les services aux entreprises se distinguent par un taux particulièrement élevé de nouveaux emplois. En Belgique, par exemple, les emplois du secteur des services aux entreprises représentaient $24,3 \%$ en 1982 et $32,5 \%$ en 1996 du total national des emplois salariés, soit une augmentation de 8,2 points de pourcentage. Alors que le nombre d'emplois dans ce secteur enregistrait une croissance, celui des secteurs primaire et secondaire chutait, passant respectivement de $3,2 \%$ et $38,9 \%$ en 1982 à $2,5 \%$ et $27,4 \%$ en 1996 , soit des baisses respectives de 0,7 et 11,5 points de pourcentage (Moyart, 2006b).

La croissance du secteur tertiaire en général et des services aux entreprises en particulier s'observe aussi au Québec. De 1976 à 2009, le nombre des emplois dans les services aux entreprises a connu une augmentation de $113 \%$, alors que les emplois $\mathrm{du}$ sous-secteur des services professionnels, techniques et scientifiques aux entreprises progressaient, eux, de $161,87 \%$. Pendant la même période, les emplois des secteurs primaire et secondaire ont chuté respectivement de $29,57 \%$ et $0,31 \%$ (tableau 1 ).

Les données de ce tableau confirment les propos de Martin (1986) selon lesquels l'économie du Québec et celle du Canada se sont tertiarisées à un rythme rapide pendant les années 1960. En 1970, selon cet auteur, le Québec était tertiarisé (en termes d'emplois) à $64,7 \%$ et le Canada à $66,6 \%$

La croissance du secteur tertiaire en général et des services aux entreprises en particulier s'observe aussi au Québec.

\subsection{Services aux entreprises et la capacité exportatrice}

Des recherches empiriques soutiennent que la capacité exportatrice des services aux entreprises est en pleine croissance depuis les années 1980 en raison des développements dans les domaines des transports et des communications. Leurs ventes 
interrégionales se chiffrent entre 20 et $50 \%$ du chiffre d'affaires des firmes prestataires, alors que leurs ventes internationales se situent entre 3 et $5 \%$. Par ailleurs, ces ventes varient suivant les villes et les régions des prestataires, les villes mondiales enregistrant plus de services aux entreprises exportés que les métropoles régionales et que les régions non métropolitaines. Il est aussi intéressant de constater que ces ventes extrarégionales ne se font pas dans une direction géographiquement unilatérale, des grandes villes vers des petites villes comme le présumait la théorie des places centrales. Des petites villes exportent aussi des services aux entreprises vers les hiérarchies supérieures et des villes de même rang exportent les unes vers les autres (Moyart, 2006b). Cependant, selon cette auteure, le pourcentage des exportations des services aux entreprises est sous-estimé, car les analyses se limitent aux exportations directes, c'està-dire aux exportations interfirmes. Or, il existe aussi des exportations intrafirmes, c'est-à-dire des transferts de services au sein de l'entreprise prestataire ou au sein de l'entreprise utilisatrice, et des exportations de services induites ou encore des exportations incorporées à un bien ou à un produit (embodied services).

\section{Il semble que les effets multiplicateurs des services aux entreprises soient plus importants qu'ils n'apparaissent à première vue.}

Ces exportations entraînent des effets multiplicateurs pour la région exportatrice : en effet, les revenus provenant de l'extérieur, lorsqu'ils sont investis ou dépensés localement, permettent de créer d'autres emplois et génèrent de nouveaux revenus à l'échelle locale. À ce sujet, il semble que les effets multiplicateurs des services aux entreprises soient plus importants qu'ils n'apparaissent à première vue. En effet, comme l'avance Laurence Moyart, le plus important pour la région n'est pas le volume du chiffre d'affaires exporté, mais la proportion des revenus d'exportation injectés et dépensés dans la région. Ainsi, une activité peut enregistrer un volume élevé d'exportations, mais un faible niveau de revenus qui restent et sont dépensés dans la région. À cet égard, les firmes prestataires des services aux entreprises se différencient du secteur industriel, car elles sont caractérisées par des liens plus étroits avec l'économie locale : elles sont généralement des entreprises locales qui utilisent la main-d'œuvre locale, consomment localement, etc. Ainsi, malgré que les services aux entreprises soient potentiellement moins exportateurs que le secteur industriel, ils injecteraient dans la région beaucoup de revenus provenant de leurs exportations et leurs effets multiplicateurs seraient égaux, voire supérieurs à ceux de l'industrie (Moyart, 2006b).

\subsection{Services aux entreprises et effet compétitivité - productivité}

$\mathrm{Si}$ la contribution directe des services aux entreprises évaluée en termes d'emplois et d'exportations est relativement facile à quantifier, leur rôle indirect est difficile, voire impossible, à mesurer, ce qui ne l'empêche pas d'avoir un impact déterminant sur le développement régional. En effet, les services aux entreprises apportent un soutien indispensable aux entreprises de tous les secteurs d'activité pour rehausser leur capacité d'innovation, leur compétitivité, leur productivité, etc. Ils se présentent comme des infrastructures immatérielles dont ont besoin des entreprises régionales et créent ainsi un environnement favorable pour les affaires dans la région (Illeris, 2006). De ce fait, les services aux entreprises sont absolument nécessaires pour maintenir et renforcer la compétitivité de la région (Martin, 1986). On retrouve ce rôle indirect des services aux entreprises partout dans le système de production, que ce soit en amont (par des services de R-D, d'études de marché, etc.), parallèlement au processus de production (services de conseil, de gestion, etc.) ou en aval (par des services de distribution, de maintenance, etc.). C'est ce qui incite Moyart (2006b) à avancer que, sans services aux entreprises, le système productif serait lourdement entravé dans son fonctionnement. Un raisonnement similaire conduit Bourgain, Catin et Pieretti (2006) à soutenir que le secteur financier, en tant que service supérieur, doit s'appuyer sur une qualité hors pair de l'information pour prendre ses décisions, ce qui permet une bonne allocation du capital et contribue à réduire le risque pour chaque entreprise qui investit, le résultat étant une meilleure efficacité des entreprises clientes.

\subsection{Services aux entreprises et attractivité régionale}

Les services aux entreprises ont la capacité d'attirer une main-d'œuvre hautement qualifiée et à salaire 
élevé, ouverte au changement et aux innovations, ce qui peut favoriser l'attractivité des régions (Moyart, 2006b). De plus, en apportant aux entreprises un soutien indispensable à leur productivité et leur compétitivité, ils créent un environnement favorable aux affaires dans les régions où leur présence est sensible et contribuent à l'établissement de nouvelles entreprises dans différents secteurs d'activité. Ainsi, Zuliani (1995, cité par Moyart, 2006b, p. 170), dans son étude sur l'agglomération toulousaine, avance que la moitié des services aux entreprises analysés ${ }^{2}$ contribuent à l'instauration d'un climat favorable aux affaires. Cet auteur constate également que, si la présence de certains services de haut niveau tels que les services informationnels, les services informatiques, les renseignements commerciaux, etc. joue un rôle déterminant dans les choix de localisation des entreprises, il arrive même que certains services banals, tels que les services bancaires, la maintenance, la restauration d'entreprise, les services de messagerie rapide, la reprographie ou encore les services de secrétariat, exercent une attraction tangible et, dans la concurrence interurbaine, parviennent à orienter certaines entreprises dans leurs décisions de s'établir ou non dans l'agglomération. Cette capacité des services aux entreprises à influencer la localisation des autres agents économiques est si importante que plusieurs auteurs la considèrent comme plus stratégique que leur potentiel d'exportation ou leur contribution directe à l'emploi (Moyart, 2006b). Aussi, en raison de leur effet attracteur, les services aux entreprises ne doivent plus être considérés comme des activités induites car « une activité qui permet d'en attirer d'autres peut véritablement être considérée comme une cause première du développement et représente clairement le contraire d'une activité induite » (Moyart, 2006b, p. 170; tiré de Polèse, 1974, p. 489).

\section{En raison de leur effet attracteur, les services aux entreprises ne doivent plus être considérés comme des activités induites.}

\subsection{Services aux entreprises et reconversion régionale}

Grâce à leur rôle dans l'accroissement de l'attractivité régionale, les services aux entreprises contribuent à la transformation, à la modernisation et à la diversification de l'économie locale et régionale. De ce fait, la reconversion de certaines régions à l'économie périclitante en est facilitée. Inversement, l'absence ou l'insuffisance de services aux entreprises dans une région ne peut qu'accentuer les difficultés qu'elle traverse. En particulier, cela se manifeste dans :

la limitation de l'accès des firmes régionales à l'innovation, le détournement de la demande régionale vers d'autres régions mieux dotées en services, l'inflation des coûts induite par ce recours accru aux prestataires extrarégionaux, le freinage des capacités locales de maîtrise de processus de développement ou encore la difficulté à retenir au niveau local la main-d'œuvre qualifiée (Moyart, 2006b, p. 169).

\section{Si les services aux entreprises sont en général reconnus pour toutes ces contributions, les SSE le sont de manière particulière.}

Si les services aux entreprises sont en général reconnus pour toutes ces contributions, les SSE le sont de manière particulière. Comme le soutiennent plusieurs auteurs, dont Martin (1986) et Polèse (1986), le tertiaire supérieur n'est pas le seul segment des services aux entreprises à dynamiser la région, mais il est celui qui a l'effet le plus important. En termes d'emplois par exemple, outre le fait de connaître une croissance plus soutenue que les autres secteurs d'activité (voir tableau 1), les SSE constituent une source substantielle d'emplois requérant une main-d'œuvre hautement qualifiée et à salaire élevé (Camacho et Rodriguez, 2006; Moyart, 2006b). Or, les ressources humaines hautement qualifiées constituent justement un facteur essentiel du développement économique. De plus, comme les entreprises évoluent dans un environnement intensément concurrentiel en raison de la libéralisation des marchés et de la mondialisation de l'économie, elles doivent se tourner vers des prestataires de SSE qui ont l'expertise nécessaire pour répondre à leurs besoins en diverses matières : fournir des connaissances sur les marchés étrangers; aider à prendre des décisions rapides et efficaces en ce qui concerne la fabrication des produits rencontrant les attentes des clients; offrir un appui constant dans la réalisation d'innovations portant sur les produits, les procédés ou la gestion; guider dans l'obtention et le traitement efficace d'une multitude d'informations disponibles (Moyart, 2006a). 


\section{DISPARITÉS RÉGIONALES EN MATIÈRE DE DÉVELOPPEMENT DES SSE}

$\mathrm{Si}$, par leur apport direct et indirect, les SSE contribuent de façon cruciale au développement des régions, leur déploiement dans l'espace est loin d'être uniforme. Les SSE, à l'instar des autres activités tertiaires, ne connaissent pas le même degré d'amplitude et de diversité d'une région à l'autre. Plusieurs chercheurs notent qu'ils se développent davantage dans des régions métropolitaines au détriment des régions non métropolitaines (Nagy, 2006; Illeris, 2006; Polèse, 1986). Dans son étude sur les services avancés aux entreprises en Hongrie, Erika Nagy (2006) constate que le développement des ces services a engendré une dichotomie métropole/campagne et zone rurale/zone urbaine. Selon elle, l'avantage de la capitale dans le secteur des services avancés aux entreprises se manifeste de plusieurs façons, dont :

la variété de services offerts, la centralisation des sièges des sociétés qui proposent ces services, en particulier celles qui sont au centre des flux de capitaux, de savoir et d'information susceptibles d'améliorer l'avantage concurrentiel (R-D, conseils financiers et techniques, publicité et relations publiques, services de logiciels spécialisés, ....), [...] la présence de l'expertise en gestion de l'information sur les marchés internationaux et des relations d'affaires (Nagy, 2006, p. 313).

D'une part, note encore Nagy, les services avancés aux entreprises ont une présence plus affirmée dans les régions dont des principaux centres comptent plus de 100000 habitants, principalement en raison de leur offre de main-d'œuvre qualifiée et de services publics (tels l'enseignement supérieur et l'administration publique). D'autre part, les régions considérées comme périphériques en termes d'accessibilité et comme à la traîne sur le plan du développement économique accusent aussi un retard du développement des services avancés aux entreprises.

En analysant des données statistiques sur l'emploi de 1982 à 2000 au Danemark et de 1989 à 1998 en France, Sven Illeris (2006) constate, pour sa part, que les emplois du secteur des services aux entreprises et du tertiaire en général connaissent une forte augmentation alors que les emplois des secteurs primaire et secondaire diminuent ou stagnent, d'une part, et que les emplois du secteur tertiaire sont plus concentrés dans des grandes villes, d'autre part. Ces disparités régionales en matière de développement des services aux entreprises se vérifient aussi au Québec. Selon Mario Polèse (1986) qui s'appuie sur certaines recherches de l'Institut national de la recherche scientifique (INRS) au Québec, le tertiaire supérieur a une tendance plus forte à se concentrer dans un milieu urbain que d'autres activités. Les résultats de notre recherche dans la région de la CapitaleNationale du Québec confirment également cette situation (voir plus bas).

Considérant ces disparités ainsi que le rôle des SSE dans le développement économique, la question qui se pose est de savoir jusqu'à quel point le développement de ces services soutient ou contrarie le développement économique des différents territoires. En d'autres mots, les régions bien dotées en SSE sont-elles économiquement plus développées et vice versa? Nous tentons d'apporter quelques éléments de réponse en étudiant le cas de la région de la Capitale-Nationale du Québec.

Le tertiaire supérieur a une tendance plus forte à se concentrer dans un milieu urbain que d'autres activités.

\section{DISPARITÉS RÉGIONALES EN MATIÈRE DE DÉVELOPPEMENT DES SSE}

La région de la Capitale-Nationale du Québec est l'une des dix-sept régions administratives du Québec. Elle est située sur la rive nord du fleuve Saint-Laurent. Elle englobe la ville de Québec et le territoire rural qui l'environne. Selon les indicateurs usuels, sa santé économique dépasse la moyenne de l'ensemble québécois. Elle se situe parmi les régions québécoises où le pourcentage d'emploi dans le secteur tertiaire est le plus élevé (Ministère du développement économique, de l'innovation et de l'exportation [MDEIE], 2006). Cette région est composée de sept territoires, soit six MRC 
(municipalités régionales de comté) et un territoire à compétence de MRC, la ville de Québec en l'occurrence. La ville de Québec est considérée comme une grande ville en raison de sa taille qui est 525380 habitants (Institut de la statistique du Québec [ISQ], 2006). Les six MRC ne comptent ensemble que 109270 habitants selon les données de l'ISQ et sont considérées comme rurales. Notre étude sur le développement des SSE dans cette région nous a permis de dresser un état de la situation dans les sept territoires de la région-territoires qui se trouvent à des niveaux différents de développement économique- et d'envisager de nouvelles perspectives pour un meilleur avenir de ces services et de l'ensemble de la région.

Pour réaliser cette recherche, nous avons, dans un premier temps, procédé à une analyse de la concentration des services aux entreprises dans la région en utilisant des données statistiques portant sur les entreprises de cinq employés et plus en activité dans la région. Nous avons trouvé ces données sur le site internet du ministère de l'Emploi et de la Solidarité sociale du Québec (MESS), http://imt.emploiquebec.net, qui héberge la liste de toutes les entreprises ayant cinq employés et plus œuvrant au Québec, et ce, par région et par domaine d'activité. Nous avons consulté ce site en novembre 2008 et obtenu une liste de 5960 entreprises faisant affaire dans cette région, et ce, dans les trois grands secteurs d'activité. Dans un deuxième temps, nous avons effectué au moyen d'un sondage par questionnaire une étude des lieux où les établissements de tous types de la région s'approvisionnent en services aux entreprises. Un échantillon cible de 735 établissements a été tiré au hasard représentant un pourcentage de $14 \%$ de la population des établissements dans les secteurs primaire et secondaire et dans le sous-secteur des services aux entreprises et $7 \%$ dans le sous-secteur du commerce de détail. Autant d'exemplaires du questionnaire ont été envoyés par la poste. Cependant, les exemplaires qui nous ont été retournés bien remplis ont été trop peu nombreux et nous avons par la suite choisi de contacter les participants par téléphone. $\mathrm{Vu}$ que l'objectif principal de ce sondage était d'étudier le développement qualitatif des services aux entreprises, les appels téléphoniques ont principalement été ciblés sur les établissements de 20 employés et plus. Au total, nous avons rassemblé 299 exemplaires du questionnaire bien remplis, dont 237 exemplaires dans la catégorie des entreprises de 20 employés et plus et 62 exemplaires dans la catégorie des entreprises de moins de 20 employés.

Comme la population d'établissements de chacune des MRC rurales de la région de la CapitaleNationale du Québec est très peu nombreuse comparativement à celle de la ville de Québec, et ce, dans tous les secteurs d'activité, les données collectées dans chacune de ces MRC sont trop faibles pour en faire une analyse par MRC et par secteur d'activité. C'est la raison pour laquelle nous avons mis l'accent sur le contraste entre la ville de Québec et l'ensemble des MRC rurales dans la présentation des résultats. Selon les tableaux 2 et 3 , les services aux entreprises, et les SSE en particulier, sont plus développés dans la ville de Québec que dans les MRC rurales de la CapitaleNationale du Québec. Concernant la concentration, les secteurs secondaire et tertiaire sont de loin plus concentrés dans la ville de Québec que dans les MRC rurales de la région. Comme le montre le tableau 2, 82,3\% des établissements du secteur secondaire et $96,3 \%$ des établissements de services aux entreprises se trouvent dans la ville de Québec. Ce pourcentage reste quasiment le même dans le cas des SSE (tableau 3).

L'hyperconcentration des activités économiques en général et des services aux entreprises en particulier dans la ville de Québec s'explique par le fait que, à la suite des réorganisations municipales de 2002 et 2006, la ville de Québec regroupe aujourd'hui la plus grande partie du territoire urbanisé de la région, soit le territoire de la ville de Québec tel qu'il existait avant 2002 et la plupart des municipalités de banlieue qui l'entouraient ${ }^{3}$.

L'étude des lieux où les établissements de la région de la Capitale-Nationale du Québec s'approvisionnent en services aux entreprises montre d'abord que, dans l'ensemble, ceux-ci achètent les trois quarts de leurs services dans la ville de Québec, alors que leurs achats dans les MRC rurales de la région ne dépassent pas $10 \%$ en moyenne (tableau 4). En deuxième lieu, on constate que ces établissements consomment plus de services provenant de Montréal que de partout ailleurs dans la province, dans le pays et dans le monde. Enfin, 
on note qu'ils achètent très peu de services en dehors de la province, c'est-à-dire ailleurs au Canada y compris la métropole de Toronto (en deçà de $5 \%$ ) et à l'étranger (en deçà de $2 \%$ ).

En distinguant les entreprises de cette région selon le nombre d'employés, on remarque que les entreprises de moins de vingt employés achètent plus souvent des services dans les MRC rurales de la région que celles de vingt employés et plus. Par contre, ces dernières achètent plus de services à Montréal que celles de moins de vingt employés. En revanche, leurs achats de services ailleurs au Québec, au Canada et à l'étranger sont dans le même ordre et sont de moindre importance.

Lorsqu'on sépare les établissements de la ville de Québec de ceux des MRC rurales de la région, on remarque que les lieux d'approvisionnement des services aux entreprises par des établissements de la ville de Québec ne diffèrent pas de manière très sensible des observations précédentes. Par ailleurs, on constate que ceux des MRC rurales de la région achètent davantage des services au niveau local. Par contre, ces établissements achètent eux aussi en moyenne plus de la moitié à l'extérieur de ces MRC, dont la grande partie (plus de $37 \%$ ) provient de la ville de Québec, une situation qui s'accentue dans le cas des établissements de vingt employés et plus. L'analyse des lieux où les établissements de la région s'approvisionnent en SSE conduit pratiquement aux mêmes observations (tableau 5) que celles qui s'appliquaient aux approvisionnements en services aux entreprises en général, tels qu'ils figurent au tableau 4. Les SSE sont en général achetés à environ $75 \%$ dans la ville de Québec, à un peu plus de $7 \%$ dans les MRC rurales et à $18 \%$ à l'extérieur de la région. Il faut remarquer par ailleurs que, d'une part, les établissements de la ville de Québec n'achètent aucun de ces services dans les MRC rurales de la région et que, d'autre part, les établissements se trouvant dans ces MRC les achètent plus dans la ville de Québec que dans ces mêmes MRC. Ceci indique que les SSE sont, tout comme les services aux entreprises en général mais sur une échelle encore plus grande, plus développés dans la ville de Québec que dans les MRC rurales de la région.

\section{DÉVELOPPEMENT ÉCONOMIQUE DANS LA RÉGION DE LA CAPITALE-NATIONALE DU QUÉBEC}

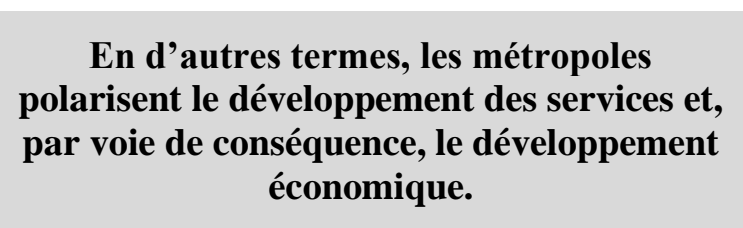

En résumé, les résultats de notre enquête sur les lieux où les établissements de la région de la Capitale-Nationale du Québec qui s'approvisionnent en services aux entreprises montrent que ceux-ci achètent la grande majorité de ces services en général, et des SSE en particulier, dans la ville de Québec et très peu de services dans les MRC rurales de la région et à l'extérieur de la région. Cela a été observé dans toutes les catégories de ces établissements selon leurs tailles. Ceci signifie que les SSE et les services aux entreprises en général se trouvant dans la ville de Québec rencontrent bien les besoins des établissements de la région et sont de loin plus développés que ceux offerts dans les MRC rurales de la région.

Considérant qu'en moyenne il y a moins de $9 \%$ des services aux entreprises et moins de $8 \%$ des
SSE qui proviennent des MRC rurales de la région, on peut sans doute dire que les services aux entreprises en général et les SSE en particulier se trouvant dans ces MRC sont sousdéveloppés. Ceci est davantage mis en lumière par le fait que les établissements de vingt employés et plus y achètent deux fois moins de services que les établissements de moins de vingt employés. Cela indique que plus les établissements de la région sont grands, moins ils préfèrent les services offerts dans ces MRC rurales. Le sous-développement des SSE dans ces MRC est aussi mis en évidence par le fait qu'aucun d'eux n'y est acheté, même dans une très faible proportion, par les établissements se trouvant dans la ville de Québec.

Ces observations corroborent les données statistiques portant sur la concentration des SSE dans la région de la Capitale-Nationale du Québec (tableau 3). Ceci renforce le constat selon lequel ces services sont de loin plus développés dans la ville de Québec que dans les MRC rurales 
de la région. Ceci nous ramène à la question posée ci-haut, à savoir si ces disparités régionales en matière de développement des SSE sont synonymes de disparités dans le développement économique. Selon Illeris (2006), puisque les services sont concentrés dans des grands pôles urbains comparativement aux autres activités économiques et qu'ils ont connu au cours des dernières décennies et connaissent toujours des taux de croissance supérieurs à ceux des autres activités, il s'ensuit que le développement économique est beaucoup plus concentré dans des grandes régions urbaines. En d'autres termes, les métropoles polarisent le développement des services et, par voie de conséquence, le développement économique. En comparant la ville de Québec et les MRC rurales de la région, on s'aperçoit aussi que plusieurs données économiques vont dans le sens d'une telle hypothèse. Si l'on regarde par exemple la concentration des activités économiques dans la région de la Capitale-Nationale du Québec (tableau 2), on remarque que tous les secteurs d'activité, sauf le secteur primaire, sont hyperconcentrés dans la ville de Québec.

L'industrialisation d'une région est l'une des caractéristiques importantes du développement économique de la région, et peut éclairer le niveau du développement économique de la ville de Québec par rapport à celui des MRC rurales de la région. Marc-Urbain Proulx (2002), dans son ouvrage intitulé L'économie des territoires au Québec: Aménagement, gestion, développement, a comparé le positionnement des territoires MRC du Québec en se basant sur le nombre des entreprises manufacturières œuvrant dans le territoire. Suivant ce critère, l'auteur a classé les MRC du Québec en trois catégories. Par ailleurs, l'auteur a étudié d'autres indices qui décrivent le soutien au développement afin de cerner les caractéristiques des territoires regroupés dans chaque catégorie. Les indices utilisés par l'auteur dans cet exercice sont l'entrepreneuriat, la formation professionnelle, l'animation socioéconomique, le nombre d'employés en R-D et le réseautage (Proulx, 2002 : 258). Cet exercice est intéressant dans ce sens qu'il permet non seulement de comparer des territoires selon leur niveau d'industrialisation, mais aussi de saisir les particularités de la place qu'ils occupent dans l'ensemble et, par extension, d'arriver à une meilleure compréhension du développement territorial. Dans le classement de Marc-Urbain Proulx, la catégorie I comprend des territoires MRC qui ont peu d'entreprises manufacturières (42 entreprises manufacturières ou moins). Selon l'auteur, les territoires de cette catégorie se caractérisent largement par la dominance du secteur primaire. Aussi, sont-ils "généralement bien pourvus en capacité innovatrice, en entrepreneuriat et en animation socioéconomique mais faiblement en réseautage » (Proulx, 2002 : 258). La catégorie II comprend des territoires MRC ayant entre 43 et 148 entreprises manufacturières. Ces territoires se caractérisent par un niveau moyen de formation professionnelle et d'entrepreneuriat et affichent par conséquent un indice moyen de potentiel de développement. La catégorie III comprend des territoires MRC dont le total des entreprises manufacturières est de 149 ou plus. Ces territoires ont un niveau avancé d'industrialisation et « sont mieux pourvus en R-D, en formation et en entrepreneuriat » (Proulx, $2002: 262$ ).

Dans la logique de cette classification et suivant la concentration du secteur secondaire dans la région de la Capitale-Nationale du Québec (tableau 2 et Proulx, 2002 : 260-265), seule la ville de Québec se trouve dans la catégorie III, soit celle qui correspond à un niveau avancé d'industrialisation. La MRC de Portneuf, qui compte 92 établissements manufacturiers, se situe dans la catégorie II, ce qui équivaut à bénéficier d'un niveau moyen d'industrialisation, alors que toutes les autres MRC appartiennent à la catégorie I. Ainsi, dans la mesure où le niveau d'industrialisation peut-être retenu comme un des critères de comparaison du niveau de développement économique, la ville de Québec est économiquement plus développée que les MRC rurales de la région de la Capitale-Nationale du Québec. Il est par ailleurs important de remarquer, comme la deuxième section de ce texte le montre, que les services aux entreprises en général, et les SSE en particulier, jouent un rôle de premier plan dans la fourniture des soutiens à l'industrialisation qui sont, entre autres et selon les indices utilisés par cet auteur, l'entrepreneuriat, la formation professionnelle, l'animation socioéconomique, la R-D et le réseautage. 


\section{CONCLUSION}

\section{Dans ce sens, les politiques devraient fortement favoriser le développement de ces services dans la perspective d'une stratégie de développement régional.}

Les services aux entreprises en général et les SSE en particulier jouent incontestablement un rôle important dans la performance des entreprises et dans le développement régional. Considérant leurs multiples et importantes contributions, les SSE apparaissent aux yeux de plusieurs comme une condition sine qua non du développement régional. Sur ce plan, la région de la Capitale-Nationale bénéficie d'une dotation avantageuse en services. Il lui est possible d'améliorer sa situation en encourageant l'augmentation de la qualité des services existants et en stimulant le développement de certains des services que les entreprises de la région doivent encore se procurer à l'extérieur. Le fait de profiter des meilleurs SSE favorise le développement des entreprises et, par le fait même, stimule le développement régional (Liefooghe, 2006). C'est pourquoi les politiques publiques devraient considérer les SSE et les services aux entreprises en général comme des activités motrices du développement régional plutôt que de les traiter comme des activités induites. Dans ce sens, les politiques devraient fortement favoriser le développement de ces services dans la perspective d'une stratégie de développement régional. Comme l'a bien dit Fernand Martin (1986), cela ne signifie pas que les autres industries ne sont pas importantes et une telle politique inclurait certainement des considérations sur ces autres industries.

Si la situation de la région de la Capitale-Nationale est somme toute assez enviable, cela ne doit pas faire oublier que, dans cette région comme dans bien d'autres, les services aux entreprises sont concentrés dans le noyau métropolitain, alors que les zones rurales demeurent plutôt mal desservies. Ces dernières font figure de parents pauvres. Bien qu'il ne puisse y avoir une conclusion absolue, les résultats de notre étude nous indiquent que le sousdéveloppement des SSE dans la zone rurale de la région de la Capitale-Nationale et dans les régions périphériques en général nuit à leur développement. Pour autant, nous pensons que le potentiel d'innovation des entreprises qui y sont situées reste important. Le simple fait d'être situé à l'écart du noyau métropolitain n'est pas un obstacle insurmontable. La capacité innovatrice des entreprises dépend davantage de leur maillage avec les partenaires stratégiques qui leur apportent les informations indispensables à leur fonctionnement que de leur proximité physique avec ces partenaires (Côté, 2005). Diverses initiatives peuvent favoriser le développement des zones rurales, même si leur dotation en services aux entreprises est moins favorable que celle du noyau métropolitain.

La première de ces initiatives est certes d'augmenter l'offre des services aux entreprises dans les zones rurales. Le potentiel d'amplification de l'offre de services demeure modeste, mais il est réel. Les entreprises de la zone rurale, surtout les plus petites, ont démontré leur propension à acheter les services locaux quand ils sont disponibles. L'augmentation de la disponibilité des services locaux devrait se traduire par une consommation plus assidue de ces mêmes services. Un effort de sensibilisation semble cependant nécessaire. Le lien entre le développement local et la disponibilité des services aux entreprises n'est pas nécessairement perçu directement par les intervenants locaux. Une simple vérification du contenu des Plans d'action pour l'économie et l'emploi (PALÉE), des Centres locaux de développement (CLD), des territoires ruraux de la région de la Capitale-Nationale de même que du contenu des documents de Planification stratégique des MRC de la région, montre que le développement des services aux entreprises ne fait pas partie des objectifs explicites de ces collectivités locales.

Une autre initiative stratégique serait de mettre en place dans les zones rurales, par exemple au sein des CLD, des formules d'intermédiation facilitant le contact avec les SSE importants pour le devenir des entreprises locales, particulièrement quand il s'agit de soutenir l'innovation dans ces entreprises, et ce, peu importe que les services visés soient présents ou non dans la MRC ou l'environnement immédiat de l'entreprise qui en a besoin. Cette façon de trouver les soutiens nécessaires à l'innovation, par exemple en matière de services de R-D, est déjà pratiquée spontanément dans les territoires non métro- 
politains par les entreprises les plus débrouillardes qui trouvent à l'extérieur de leur milieu les partenaires stratégiques essentiels à leur développement (Côté, 2002).

En combinant l'augmentation des services disponibles au niveau local et l'intermédiation pour effectuer le contact avec les services extérieurs qui ont une valeur stratégique pour les entreprises locales, les zones rurales conserveraient une capacité d'innovation apte à stimuler leur développement malgré une dotation qui demeurera toujours plus faible que celle du noyau métropolitain.

\section{BIBLIOGRAPHIE}

Bourgain A., Catin M. et Pieretti P., (2006), «Pôle financier et croissance régionale : les effets externes des activités bancaires sur l'économie luxembourgeoise », in Gallouj C. et al., (eds), Services aux entreprises et développement régional : bilan et perspectives, Éditions de Boeck Université, Bruxelles, pp. 341-363.

Browning H. L. et Singelmann J., (1978), «The Transformation of the US Labor Force : the Interaction of Industry and Occupation », Politics and Society, vol. 8, $\mathrm{n}^{\circ}$ 3-4, pp. 481-509.

Camacho J. A. et Rodriguez M., (2006), «Services et développement régional », in Gallouj C. et al., (eds), Services aux entreprises et développement régional: bilan et perspectives, Éditions de Boeck Université, Bruxelles, pp. 397-416.

Côté S., (2005), "Vers un modèle de soutien à l'innovation pour les entreprises québécoises », in Jean B. et Lafontaine D., (eds), Territoires et fonctions, tome 2, Des pratiques aux paradigmes : les systèmes régionaux et les dynamiques d'innovation en débats, Éditions du GRIDEQ et Éditions du CRDT, collection Tendances et débats en développement régional, $\mathrm{n}^{\circ}$ 9, Rimouski, pp. 25-40.

Côté S., (2002), «L'enjeu de l'innovation pour les régions québécoises », in ouvrage collectif sous l'égide du Mouvement Territoire et Développement, Le développement des territoires: nouveaux enjeux, UQAR-GRIDEQ, collection Actes et instruments de la recherche en développement régional, no 14, Rimouski, pp. 95-105.

Dansereau F. et Polèse M., (1977), Étude du tertiaire privé dans les agglomérations de l'Abitibi Témiscamingue. Dossier d'inventaire et d'analyse, l'Office de planification et de développement du Québec, collection Les schémas régionaux, Québec.

Fourastié J., (1949), Le grand espoir du XX $\mathrm{XX}^{\mathrm{e}}$ siècle : progrès technique, progrès économique, progrès social, Presses universitaires de France, Paris.
Fuchs V. R., (1968), The Service Economy, Columbia University Press, New York.

Gallouj C., Leloup F., Mérenne-Schoumaker B. et Moyart L., (eds), (2006), Services aux entreprises et développement régional : bilan et perspectives, Éditions de Boeck Université, Bruxelles.

Illeris S., (2006), «Quels rôles pour les services dans le développement régional? », in Gallouj C. et al., (eds), Services aux entreprises et développement régional: bilan et perspectives, Éditions de Boeck Université, Bruxelles, pp. 47-64.

Institut de la statistique du Québec, (2006), Recensement de la population, Population selon le groupe d'âge, région administrative de la Capitale-Nationale. http://www.stat.gouv.qc.ca/regions/recens2006_03/popu lation03/poptot03.htm. Consulté le 22 mars 2011.

Institut de la statistique du Québec (ISQ), (2010), Enquête sur la population active. Disponible sur Internet : http://www.stat.gouv.qc.ca/donstat/societe/mar ch_travl_remnr/cat_profs_sectr_activ/professions/pop_a ctive. Consulté le 5 janvier 2011.

Liefooghe C., (2006), «Système territorial et sousdéveloppement des services aux entreprises : analyse des freins au développement des services aux entreprises dans l'ancien bassin minier du Nord-Pas-de-Calais », in Gallouj C. et al., (eds), Services aux entreprises et développement régional : bilan et perspectives, Éditions de Boeck Université, Bruxelles, pp. 210-234.

Martin F., (1986), «Le rôle du secteur tertiaire dans la stratégie de développement économique », in Savoie D. J. et Raynauld A., (eds), Essais sur le développement régional, Presses de l'Université de Montréal, Montréal, pp. 123-134.

Martinelli F., (1991), «Services aux producteurs et développement régional », Espaces et Sociétés, nº 66-67, pp. 185-216.

Ministère de l'emploi et de la solidarité sociale du Québec (MESS), (2008), Trouver une entreprise. Disponible au http://imt.emploiquebec.net, consulté en novembre 2008.

Ministère du développement économique, de l'innovation et de l'exportation (MDEIE), (2006), Portrait socioéconomique des régions du Québec, Québec.

Moyart L., (2006a), «De la croissance tertiaire à la croissance des services aux entreprises : une synthèse des théories et modèles explicatifs », in Gallouj C. et al., (eds), Services aux entreprises et développement régional: bilan et perspectives, Éditions de Boeck Université, Bruxelles, pp. 17-45.

Moyart L., (2006b), «Le rôle des services aux entreprises dans le développement régional : quelles leçons en tirer pour les villes moyennes en Belgique? », in Gallouj C. et al., (eds), Services aux entreprises et développement régional: bilan et perspectives, Éditions de Boeck Université, Bruxelles, pp. 161-187. 
Nagy E., (2006), « Développement régional et essor du secteur des services avancés aux entreprises dans une économie de marché émergente », in Gallouj C. et al., (eds), Services aux entreprises et développement régional: bilan et perspectives, Éditions de Boeck Université, Bruxelles, pp. 289-318.

Organisation de coopération et de développement économiques, (1999), les services stratégiques aux entreprises, Éditions de l'OCDE, Paris.

Polèse M. et Shearmur R., (2005), Économie urbaine et régionale : introduction à la géographie économique, $2 \mathrm{e}$ éd., Economica, Paris.

Polèse M., (1974), «Le secteur tertiaire et le développement économique régional : vers un modèle opérationnel des activités motrices », L'actualité économique, pp. 475-490.

Polèse M., (1986), «Le secteur tertiaire et le développement économique régional : vers un modèle opérationnel des activités motrices », in Savoie D. J. et Raynauld A., (eds), Essais sur le développement régional, Presses de l'Université de Montréal, Montréal, pp. 135150.

Proulx M.-U., (2002), L'économie des territoires au Québec: Aménagement, gestion, développement, Les Presses de l'Université du Québec, Québec.

Shearmur R., (2010), «Space, Place and Innovation : a Distance-Based Approach », Le géographe canadien / The Canadian Geographer, vol. 54, $n^{\circ}$ 1, pp. 46-67.

Shearmur R. et Doloreux D., (2009), «Place, Space and Distance: Towards a Geography of KnowledgeIntensive Business Services Innovation », Industry and Innovation, vol. 16, $\mathrm{n}^{\circ} 1$, pp. 79-102.

Zuliani J. M., (1995), Les services aux entreprises dans une métropole à forte composante de recherchedéveloppement: Toulouse, 5e Conférence annuelle du RESER, Aix-en-Provence.

\section{NOTES}

${ }^{1}$ Sujet d'une thèse de doctorat en développement régional, la recherche porte sur les liens entre les services aux entreprises et le développement régional et effectue une comparaison entre la région administrative de la Capitale-Nationale au Québec et la région urbaine et rurale du grand Kigali au Rwanda.

${ }^{2}$ Transport et entreposage, ingénierie et études techniques, informatique, publicité, conseils et études, analyses et contrôles techniques, comptabilité, nettoyage, restauration d'entreprises, sécurité, secrétariat, traduction, documentation, reprographie.

${ }^{3}$ Plusieurs municipalités de l'ancienne Communauté Urbaine de Québec ont fusionné avec la ville de Québec, soit Cap-Rouge, Sainte-Foy, Sillery, Notre-Dame-des-Anges, Vanier, Québec, Val-Bélair, Loretteville, Saint-Émile, Lac-Saint-Charles, Charlesbourg et Beauport. Seules les municipalités de SaintAugustin-de-Desmaures et de L'Ancienne-Lorette ont décidé de ne pas faire partie de cette fusion. 
ANNEXE 1 - TABLEAUX

Tableau 1 - Évolution de l'emploi au Québec par grands secteurs et par sous-secteurs dans le cas des services entre 1976 et 2009

\begin{tabular}{|c|c|c|c|c|c|c|}
\hline \multirow[t]{2}{*}{ GRANDS SECTEURS } & \multicolumn{2}{|c|}{1976} & \multicolumn{2}{|c|}{2009} & \multicolumn{2}{|c|}{$\begin{array}{l}\text { Variation } \\
1976-2009\end{array}$} \\
\hline & $\mathbf{K}$ & $\begin{array}{c}\% \\
\text { emploi } \\
\text { total }\end{array}$ & $\mathbf{K}$ & $\begin{array}{c}\% \\
\text { emploi } \\
\text { total }\end{array}$ & $\mathbf{K}$ & $\%$ \\
\hline $\begin{array}{l}\text { SECTEUR PRIMAIRE } \\
\text { (agriculture, foresterie, pêche, } \\
\text { mines et extraction de pétrole } \\
\text { et de gaz) }\end{array}$ & 124,1 & 4,88 & 87,4 & 2,27 & $-36,7$ & $-29,57$ \\
\hline $\begin{array}{l}\text { SECTEUR SECONDAIRE } \\
\text { (fabrication et construction) }\end{array}$ & 745,3 & 29,30 & 742,7 & 19,32 & $-2,6$ & $-0,35$ \\
\hline $\begin{array}{l}\text { SECTEUR TERTIAIRE } \\
\text { (services) }\end{array}$ & 1674,0 & 65,82 & 3014,1 & 78,41 & $+1340,1$ & $+80,05$ \\
\hline Total des trois grands secteurs & 2543,4 & 100 & 3844,2 & 100 & $+1300,8$ & $+51,14$ \\
\hline Sous-secteurs des services & & & & & & \\
\hline * Services aux entreprises (SE) & 380,3 & 14,95 & 810,0 & 21,07 & $+429,7$ & $+112,98$ \\
\hline $\begin{array}{l}\text { [Au sein des SE : Services } \\
\text { professionnels, techniques } \\
\text { et scientifiques] }\end{array}$ & {$[190,4]$} & {$[7,49]$} & {$[498,6]$} & {$[12,97]$} & {$[+308,2]$} & {$[+161,87]$} \\
\hline $\begin{array}{l}\text { * Services aux particuliers et } \\
\text { services publics (y compris le } \\
\text { commerce de gros et le } \\
\text { commerce de détail) }{ }^{\bullet}\end{array}$ & 1293,7 & 50,84 & 2204,1 & 57,34 & $+910,4$ & $+70,37$ \\
\hline $\begin{array}{l}\text { Total du grand secteur des } \\
\text { services }\end{array}$ & 1674,0 & 65,82 & 3014,1 & 78,41 & $+1340,1$ & $+80,05$ \\
\hline \multicolumn{7}{|c|}{$\begin{array}{l}\text { e Habituellement, les services de commerce de gros sont fournis aux entreprises alors que les } \\
\text { services de commerce de détail sont en grande partie destinés aux particuliers. Ces derniers sont } \\
\text { normalement classés parmi les services aux ménages (MARTINELLI, 1991). Cependant, l'Institut } \\
\text { de la statistique du Québec (source des données de ce tableau) ne sépare pas les emplois créés } \\
\text { par le commerce de gros de ceux du commerce de détail. Ceci ne nous permet pas de savoir les } \\
\text { emplois du commerce de gros afin de les ajouter à ceux créés par les services aux entreprises, } \\
\text { ce qui les sous-estime et surestime les emplois des services aux particuliers. }\end{array}$} \\
\hline
\end{tabular}

Source : Tableau élaboré sur la base des données de l'INSTITUT DE LA STATISTIQUE DU QUÉBEC, 2010. 
Tableau 2 - Concentration des activités économiques dans la Capitale-Nationale du Québec

\begin{tabular}{|c|c|c|c|c|c|c|c|c|}
\hline \multirow[b]{2}{*}{ Établissements } & \multirow[b]{2}{*}{$\begin{array}{l}\text { Ville de } \\
\text { Québec } \\
\end{array}$} & \multirow[b]{2}{*}{$\begin{array}{l}\text { Total } \\
\text { MRC } \\
\text { rurales } \\
\end{array}$} & \multicolumn{6}{|c|}{ Détail des MRC rurales } \\
\hline & & & Charlevoix & $\begin{array}{l}\text { Charle- } \\
\text { voix-Est }\end{array}$ & \begin{tabular}{|c|}
$\begin{array}{c}\text { La Côte- } \\
\text { de- } \\
\text { Beaupré }\end{array}$ \\
\end{tabular} & \begin{tabular}{|c|} 
La \\
Jacques- \\
Cartier \\
\end{tabular} & Portneuf & $\begin{array}{c}\text { Île- } \\
\text { d'Orléans }\end{array}$ \\
\hline & $\begin{array}{r}\text { Nombre } \\
\% \\
\end{array}$ & \begin{tabular}{|l|} 
Nombre \\
\end{tabular} & $\begin{array}{r}\text { Nombre } \\
\end{array}$ & $\begin{array}{r}\text { Nombre } \\
\end{array}$ & $\begin{array}{r}\text { Nombre } \\
\%\end{array}$ & $\begin{array}{r}\text { Nombre } \\
\% \\
\end{array}$ & $\begin{array}{r}\text { Nombre } \\
\% \\
\end{array}$ & \begin{tabular}{|l} 
Nombre \\
$\%$
\end{tabular} \\
\hline Secteur primaire & 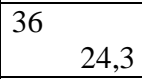 & 112 & 10 & 10 & 14 & 2,7 & $\begin{array}{ll}38 & \\
& 25,7 \\
\end{array}$ & 36 \\
\hline Secteur secondaire & $\begin{array}{r}1122 \\
82,3 \\
\end{array}$ & 241 & 38 & 30 & 44 & 21 & 92 & 16 \\
\hline \begin{tabular}{|l|}
$\begin{array}{l}\text { Services aux } \\
\text { entreprises }\end{array}$ \\
\end{tabular} & \begin{tabular}{|r|}
2401 \\
96,3 \\
\end{tabular} & 3,7 & 12 & 12 & 0,0 & 0,1 & 65 & 1 \\
\hline Commerces de détail & $\begin{array}{r}1654 \\
84,6\end{array}$ & 301 & 48 & 56 & 60 & 15 & 114 & 8 \\
\hline Total & \begin{tabular}{|r|}
5213 \\
87,5 \\
\end{tabular} & 12,5 & 108 & 108 & 119 & 42 & $\begin{array}{ll}309 & \\
& 5,2 \\
\end{array}$ & 61 \\
\hline
\end{tabular}

Source : Tableau élaboré à partir des données du MESS (2008).

Tableau 3 - Concentration des établissements de SSE dans la Capitale-Nationale du Québec

\begin{tabular}{|c|c|c|c|c|c|c|c|c|}
\hline & & & \multicolumn{6}{|c|}{ Détail des MRC rurales } \\
\hline & $\begin{array}{l}\text { Ville de } \\
\text { Québec }\end{array}$ & $\begin{array}{l}\text { Total } \\
\text { MRC } \\
\text { rurales }\end{array}$ & $\begin{array}{l}\text { Charlevoi } \\
\mathbf{x}\end{array}$ & $\begin{array}{l}\text { Charlevoi } \\
\text { x-Est }\end{array}$ & $\begin{array}{l}\text { La Côte- } \\
\text { de- } \\
\text { Beaupré }\end{array}$ & \begin{tabular}{|l|} 
La \\
Jacques \\
-Cartier \\
\end{tabular} & $\begin{array}{l}\text { Portneu } \\
\text { f }\end{array}$ & $\begin{array}{l}\text { Île- } \\
\text { d'Orléans }\end{array}$ \\
\hline $\begin{array}{l}\text { Les SSE } \\
\text { \% par rapport au total de la } \\
\text { catégorie }\end{array}$ & $\begin{array}{r}\text { Nombre } \\
\% \\
\end{array}$ & $\begin{array}{r}\text { Nombre } \\
\% \\
\end{array}$ & $\begin{array}{r}\text { Nombre } \\
\% \\
\end{array}$ & \begin{tabular}{r|} 
Nombre \\
$\%$ \\
\end{tabular} & $\begin{array}{r}\text { Nombre } \\
\%\end{array}$ & $\begin{array}{r}\text { Nombre } \\
\% \\
\end{array}$ & $\begin{array}{r}\text { Nombre } \\
\% \\
\end{array}$ & $\begin{array}{r}\text { Nombre } \\
\%\end{array}$ \\
\hline $\begin{array}{l}\text { Services de conseils en } \\
\text { gestion, conseils } \\
\text { professionnels et techniques }\end{array}$ & $\begin{array}{|ll|}47 & \\
& 97,9 \\
\end{array}$ & 2,1 & 0,0 & 0,0 & 0,0 & 0,0 & 1,0 & 0,0 \\
\hline $\begin{array}{l}\text { Services de financement, } \\
\text { d'assurance, de comptabilité } \\
\text { et vérification et services } \\
\text { connexes }\end{array}$ & $\begin{array}{r}408 \\
95,3 \\
\end{array}$ & 4,7 & 0,0 & 0,0 & 0,0 & 0,0 & 3,6 & 0,0 \\
\hline $\begin{array}{l}\text { Services d'architecture, } \\
\text { d'arpentage-géomètre, de } \\
\text { génie, de design spécialisé et } \\
\text { services connexes }\end{array}$ & 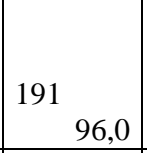 & 4,0 & 1,0 & 1,0 & 0,0 & 0,0 & 2,0 & 0,0 \\
\hline $\begin{array}{l}\text { Services informatiques et } \\
\text { services connexes }\end{array}$ & $\begin{array}{|ll|}111 & \\
& 97,4 \\
\end{array}$ & 2,6 & 0,0 & 0,0 & 0,0 & 0,0 & 2,6 & 0 \\
\hline $\begin{array}{l}\text { Services de communication et } \\
\text { services connexes }\end{array}$ & \begin{tabular}{|r|r|}
107 & \\
& 97,3 \\
\end{tabular} & 2,7 & 0,0 & 0,0 & 0,0 & 0,0 & 2,7 & 0,0 \\
\hline $\begin{array}{l}\text { Services de recherche et de } \\
\text { développement scientifique }\end{array}$ & $\begin{array}{|ll|}53 & \\
& 98,1 \\
\end{array}$ & 1,9 & 0,0 & 0,0 & 0,0 & 0,0 & 0,0 & 1 \\
\hline $\begin{array}{l}\text { Services d'études de marché } \\
\text { et de sondages d'opinion } \\
\text { (marketing) }\end{array}$ & $\begin{array}{|ll|}5 & \\
& 100,0 \\
\end{array}$ & 0,0 & 0,0 & 0,0 & 0,0 & 0,0 & 0,0 & 0 \\
\hline Services juridiques & $\begin{array}{|ll|}71 & \\
& 93,4 \\
& \end{array}$ & 6,6 & 1,3 & 1,3 & 0,0 & 0,0 & 3,9 & 0 \\
\hline $\begin{array}{l}\text { Tous les autres services } \\
\text { professionnels, scientifiques } \\
\text { et techniques }\end{array}$ & $\begin{array}{|ll|}5 & \\
& 83,3 \\
\end{array}$ & 16,7 & 0,0 & 0,0 & 0,0 & 0,0 & 16,7 & 0 \\
\hline $\begin{array}{l}\text { Total des établissements de } \\
\text { SSE. }\end{array}$ & $\begin{array}{r}998 \\
96,0 \\
\end{array}$ & 42 & 0,48 & 0,48 & 0,04 & 0,08 & $\begin{array}{ll}65 & \\
& 2,61 \\
\end{array}$ & 0,04 \\
\hline
\end{tabular}

Source : Tableau élaboré à partir des données du MESS (2008). 
Tableau 4 - Lieux de provenance des services aux entreprises pour les établissements de la région de la Capitale-Nationale du Québec (en \%)

\begin{tabular}{|c|c|c|c|c|c|c|c|c|c|c|}
\hline Catégories & $\begin{array}{l}\text { Ville de } \\
\text { Québec }\end{array}$ & $\begin{array}{l}\text { MRC } \\
\text { rurales }\end{array}$ & $\begin{array}{l}\text { Grand } \\
\text { Montréal }\end{array}$ & $\begin{array}{l}\text { Ailleurs au } \\
\text { Québec }\end{array}$ & Toronto & $\begin{array}{l}\text { Ailleurs } \\
\text { au Canada }\end{array}$ & É.U. & Europe & Internet & $\begin{array}{l}\text { Ailleurs } \\
\text { dans le } \\
\text { monde }\end{array}$ \\
\hline Ensemble & 75,05 & 8,36 & 8,23 & 2,36 & 1,87 & 2,38 & 0,89 & 0,56 & 0,11 & 0,20 \\
\hline$<20$ & 75,95 & 9,89 & 6,59 & 2,10 & 2,02 & 2,31 & 0,45 & 0,48 & 0,06 & 0,15 \\
\hline$>20$ & 73,29 & 5,38 & 11,42 & 2,87 & 1,58 & 2,52 & 1,73 & 0,71 & 0,20 & 0,31 \\
\hline \multicolumn{11}{|c|}{ Etablissements de la ville de Québec } \\
\hline Ensemble & 82,55 & 0,04 & 8,42 & 2,42 & 2,03 & 2,63 & 0,97 & 0,58 & 0,13 & 0,23 \\
\hline$<20$ & 84,59 & 0,00 & 6,77 & 2,22 & 2,37 & 2,72 & 0,52 & 0,55 & 0,07 & 0,18 \\
\hline$>20$ & 78,87 & 0,10 & 11,41 & 2,77 & 1,43 & 2,47 & 1,79 & 0,61 & 0,23 & 0,31 \\
\hline \multicolumn{11}{|c|}{ Etablissements des MRC rurales de la Capitale-Nationale du Québec } \\
\hline Ensemble & 37,60 & 49,87 & 7,25 & 2,05 & 1,09 & 1,13 & 0,46 & 0,49 & 0,00 & 0,07 \\
\hline$<20$ & 38,66 & 52,59 & 5,79 & 1,54 & 0,54 & 0,53 & 0,18 & 0,18 & 0,00 & 0,00 \\
\hline$>20$ & 34,54 & 41,98 & 11,47 & 3,53 & 2,68 & 2,87 & 1,28 & 1,40 & 0,00 & 0,26 \\
\hline
\end{tabular}

Source : Notre enquête dans la région de la Capitale-Nationale du Québec, 2009

Tableau 5 - Lieux de provenance des SSE par les établissements de la région de la CapitaleNationale du Québec (en \%).

\begin{tabular}{|c|c|c|c|c|c|c|c|c|c|}
\hline & \multicolumn{3}{|c|}{$\begin{array}{l}\text { Ensemble des établissements } \\
\text { de la région }\end{array}$} & \multicolumn{3}{|c|}{$\begin{array}{l}\text { Établissements de la ville de } \\
\text { Québec }\end{array}$} & \multicolumn{3}{|c|}{$\begin{array}{l}\text { Établissements des MRC } \\
\text { rurales de la région }\end{array}$} \\
\hline & $\begin{array}{l}\text { Ville de } \\
\text { Québec }\end{array}$ & $\begin{array}{l}\text { MRC } \\
\text { rurales }\end{array}$ & $\begin{array}{l}\text { Extra- } \\
\text { région }\end{array}$ & $\begin{array}{l}\text { Ville de } \\
\text { Québec }\end{array}$ & $\begin{array}{l}\text { MRC } \\
\text { rurales }\end{array}$ & $\begin{array}{l}\text { Extra- } \\
\text { région }\end{array}$ & $\begin{array}{l}\text { Ville de } \\
\text { Québec }\end{array}$ & $\begin{array}{l}\text { MRC } \\
\text { rurales }\end{array}$ & $\begin{array}{l}\text { Extra- } \\
\text { région }\end{array}$ \\
\hline $\begin{array}{l}\text { Emprunt pour fin } \\
\text { d'expansion }\end{array}$ & 71,00 & 7,36 & 21,64 & 76,10 & 0,00 & 23,90 & 53,42 & 32,76 & 13,82 \\
\hline $\begin{array}{l}\text { Financement des ventes à } \\
\text { tempérament }\end{array}$ & 67,67 & 5,63 & 26,70 & 70,37 & 0,00 & 29,63 & 43,06 & 56,94 & 0,00 \\
\hline $\begin{array}{l}\text { Assurances (courtiers, } \\
\text { experts en sinistre, etc.) }\end{array}$ & 64,51 & 8,74 & 26,76 & 69,18 & 0,00 & 30,82 & 38,19 & 57,88 & 3,92 \\
\hline $\begin{array}{l}\begin{array}{l}\text { Placements et valeurs } \\
\text { mobilières }\end{array} \\
\end{array}$ & 77,80 & 7,35 & 14,85 & 87,72 & 0,00 & 12,28 & 36,13 & 38,21 & 25,65 \\
\hline $\begin{array}{l}\text { Comptabilité } \\
\text { vérification }\end{array}$ & 72,87 & 10,67 & 16,46 & 83,72 & 0,00 & 16,28 & 17,06 & 65,57 & 17,37 \\
\hline $\begin{array}{l}\text { Marketing (études de } \\
\text { marché) }\end{array}$ & 69,52 & 0,96 & 29,53 & 70,20 & 0,00 & 29,80 & 58,27 & 16,73 & 25,00 \\
\hline Conseils en gestion & 79,78 & 4,19 & 16,04 & 82,46 & 0,00 & 17,54 & 64,68 & 27,75 & 7,58 \\
\hline Informatiques & 70,12 & 6,47 & 23,41 & 77,98 & 0,00 & 22,02 & 44,76 & 27,37 & 27,87 \\
\hline Communication & 75,35 & 7,16 & 17,49 & 80,11 & 0,00 & 19,89 & 43,53 & 55,04 & 1,43 \\
\hline Génie & 87,98 & 2,99 & 9,03 & 94,57 & 0,00 & 5,43 & 61,13 & 15,16 & 23,72 \\
\hline Études techniques (R-D) & 73,75 & 2,51 & 23,74 & 76,89 & 0,00 & 23,11 & 61,19 & 12,57 & 26,24 \\
\hline Services juridiques & 75,25 & 4,48 & 20,27 & 76,78 & 0,00 & 23,22 & 65,60 & 32,63 & 1,78 \\
\hline Entrepreneurs spécialisés & 74,94 & 18,24 & 6,82 & 94,16 & 0,00 & 5,84 & 29,39 & 61,46 & 9,15 \\
\hline Architecte & 69,01 & 11,92 & 19,07 & 77,11 & 0,00 & 22,89 & 55,30 & 32,11 & 12,59 \\
\hline Arpenteurs-géomètres & 66,49 & 17,46 & 16,04 & 80,08 & 0,00 & 19,92 & 24,67 & 71,23 & 4,10 \\
\hline Propriété intellectuelle & 100,00 & 0,00 & 0,00 & 100,00 & 0,00 & 0,00 & 0,00 & 0,00 & 0,00 \\
\hline Moyenne & 74,75 & 7,25 & 18,00 & 81,09 & 0 & 19,91 & 46,42 & 40,23 & 13,35 \\
\hline
\end{tabular}

Source : Notre enquête dans la région de la Capitale-Nationale du Québec, 2009 

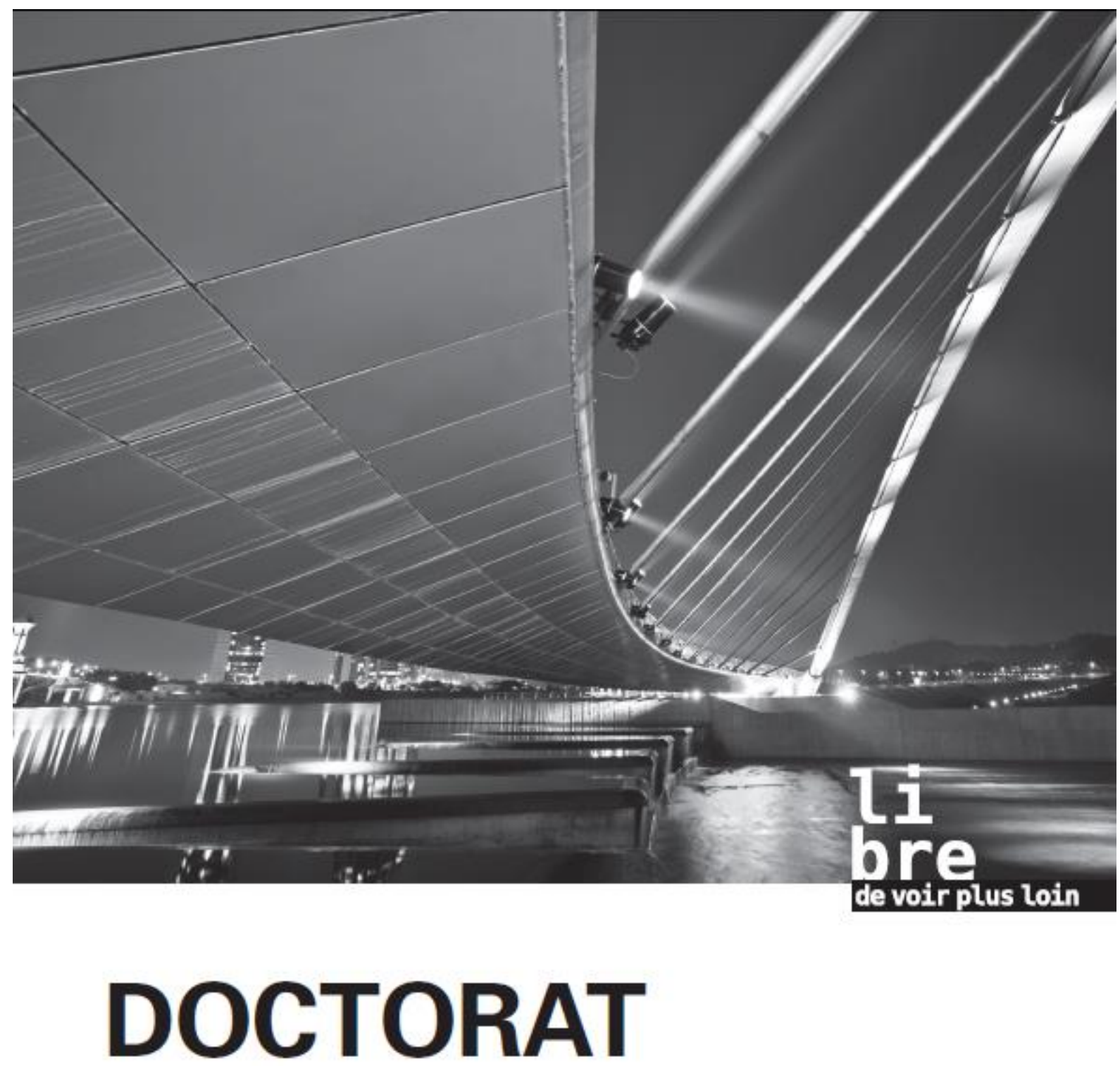

\section{EN MANAGEMENT DE PROJETS}

- Programme novateur et unique dans la francophonie

- Bourses du fonds de soutien du doctorat en management de projets

Université du Québec à Chicoutimi

418 545-5011, poste 5282

uqac.ca/dmp

uqac.ca

UQAC 